\section{Use of Concept Maps to Aid Early Engineering Design}

J.S. Weerasinghe, Ryerson University. jweerasi@ryerson.ca F.A. Salustri, Ryerson University. salustri@ryerson.c

This paper reports on the use of concept maps as a tool to facilitate innovation and aid in the communication of ideas during the initial stages of engineering design. The authors propose that engineering design, especially in the early stages, is a learning exercise. In exploring the different characteristics, requirements, and constraints of a design problem, a design team can better understand the limitations of their design, as well as encourage more
innovative thinking that works within these limitations. For engineering purposes, the organization of different innovative thinking that works within these limitations. For engineering purposes, the organization of different
ideas with concept maps may provide insight into the kinds of subsystems the design may require as well as the
interconnectivity between these subsystems as represented by the links between various nodes and the evolution of concept maps may help understand the collaborative design processes used by the designers who created the maps.

\subsection{Introduction}

Concept maps are a specific type of web diagram that facilitates the exploration, gathering and sharing of ideas. Using a medium like concept map
is a powerful method to portray a large amount of complex information, making it easier to understan and see the interactions between concepts. Novak [7] describes concept maps as a visual roadmap to show pathway that connect concepts. This makes the Paw in now in an organized manner that can be easily possible to answer focused questions about the context of the map.

Concept maps have been predominantly used in education as a tool to aid learning as well a assess understanding of what is being taugh knowledge in a way that they can understand. This also makes it easier for them to see possible relationships between different pieces of knowledge, thus further advancing their understanding. Once new knowledge is learned, the student can go back his new knowledge can be integrated into the existing
body. The early stages of engineering design can
be seen as a learning process in that each member o a design team has to become familiar with eac other's capacity as well as understand the proble that is set before them, so an educational tool may provide insight into the design process. Success depends on their ability to explain their knowledge and then represent it in such a way that it suppor effective examination by other members [1]. But
learning a piece of knowledge cannot be accomplished through reading alone, it requires
dialogue, exchange, sharing and most often compromise. It is this back and forth sharing of idea for engick cor engineering design. With concept maps a tean
can firmly establish the different aspect of thei limitations have been established, each member can

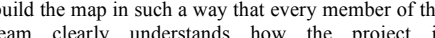
developing.

\subsection{Concept Maps}

Concept maps consist of nodes connected by links. A node is a geometric shape (usually concept, idea, item, or question. A link connects two or more concepts together, and represents relationship between the concepts. Links are labeled with a text annotation describing the nature of the elationship. The links can be unidirectional or bdirectional, depending on the nature of the
relationship. To sers can change the graphical properties of these elements. For nodes, the style can be altered to group common concepts by colour or shape or font of the node's text. For links, he style can also be altered to reflect various types of relationships between nodes, style (e.g. dashed versus solid lines). Concept maps make no specific commitments to what such style variations mean. Instead, one expects the user to establish conventions regarding style that are meaningful to the expected "readers" of the map. design problem and visually layout the constrain within which their design will fall. Once those contribute their expertise on the problem and thus
In a design setting this means that each user community can establish their own norms, thu increasing flexibility while allowing the groups to improving the capacity of the maps to convey knowledge. One useful tool that CmapTools [3], concept mapping software tool, provides is the ability to make custom styles for nodes. As mentioned earlier, the graphical elements of a node can be changed to provide added information to the map concept nodes by using a certain style pattern, he/ho may do so by creating a custom style. Once the desired combination of style elements have been selected they can be saved as a 'New Style' on the Cmap. Now instead of going to each individual node and configuring it to the one style, the user can jus style so that all of those nodes are now visully linked. This flexibility can also be added to maps that are loaded onto a server. This gives collaborative team the ability to use these custom styles from multiple locations as the style is now nked to that specific map.

Cn also also provides a server capability central location syners to view and edit maps from Synchronous collaboration allows two or more users to be logged into the server and edit the same concep map. This provides a more robust tool for collaboration as the designers can now work together tharing their knowhedge and in real time, which greatly increases the peed at which the project progresses. The added benefit of a real time chat facility to allows users to discuss the map and potential changes all within the same medium. For asynchronous collaboration, authorized users have the opportunity to view the map and make their own editions and suggestion in the map. This gives design teams that are not colocated the opportunity to collaborate on a project without having to spend large resources to bring everyone into a conference room.

\subsection{Auto21 Design Case}

An initiative called The Automobile of the $21^{\text {st }}$ Century (http://auto21.ca) was undertaken within the Canadian Network of Centres of Excellence and provides funding for a number of research projects in all aspects of automobile design among which is an
interest in collaborative design methods and tools for design teams. One such project brings together
desters number of universities to study the design
nusign to methodologies in the North American automobile industry and investigate collaborative tools that may aid in these design strategies. Among these tools concept mapping, which is used to help organize stages of an engineering project. This project provides the opportunity to observe and analyze the use of concept maps generated by members from each university to see how effective the maps are in actiltating the overall project. In order to do this part of the team will explore the design of a small will observe the design process and analyze the collaboration between the different schools. The current authors are part of the latter team.

\subsection{Concept Map Iteratio}

The initial stages of the design process in the project were very slow since all the members of the mapping software, so they were not able to use it to its full potential for collaborative work. From their lirst maps it can be seen that everyone was creating their own map, but they all provided the same basic example to evaluate how each member understands the goals of the project, it does little to provide progress as no new concepts are being generated, thus no new knowledge is being shared.

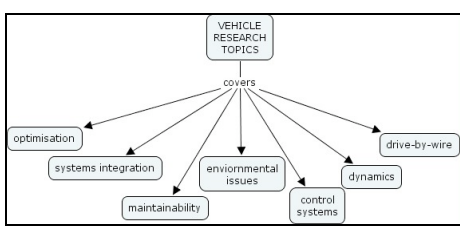

Figure 1: Initial Concept Map

Further into the project team members were developing maps with new concepts and new
knowledge based on older concept maps. While this showed definite progress in the project, there resulted For collaboration, it would be more useful for tean members to add concepts to one map and allow it to grow as new information is added thus allowing each member to contribute their expertise to the projec and see how that knowledge fits in the "big picture." Figure 1, which shows one of the initint con with maps that was created after the first meetings of the Auto21 project. Most of the nodes in this map represent general concepts requiring further 


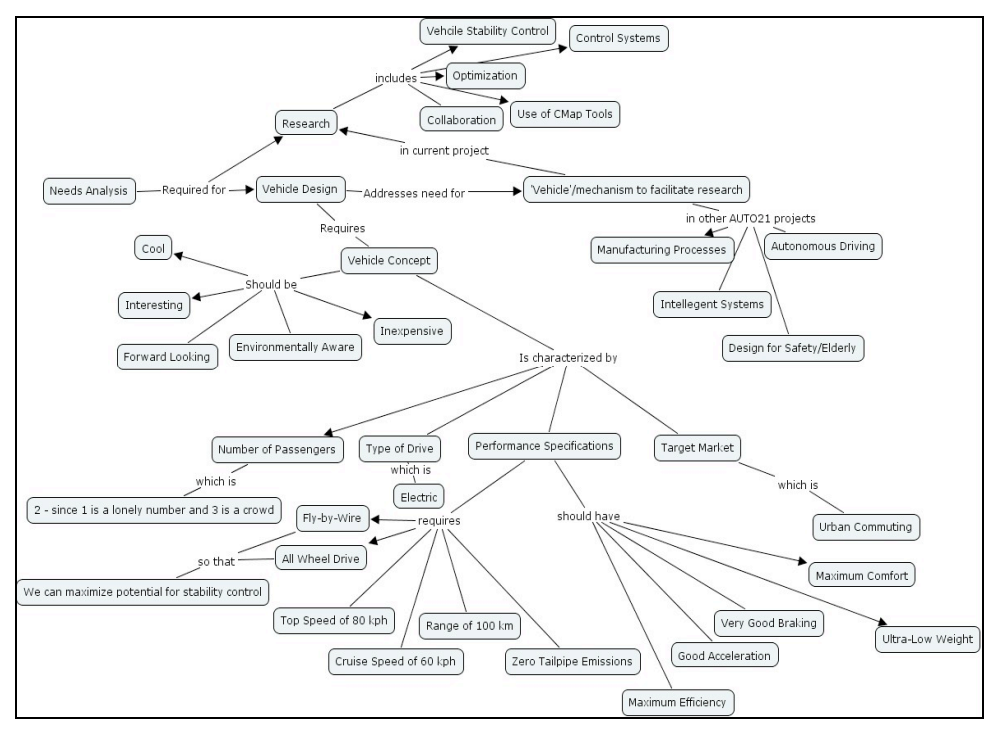

Figure 2: Project Concept Map

definition, so there is very little detail shown, but the basic information of the project could easily be learning about concept maps, so the depth knowledge represented was very low. Still, there is clear distinction between the two project stream vehicle design, and research.

Looking now to a map created a couple of months later, figure 2, we can see there has been well as addition of new concepts that were found to be relevant to the project. The two areas of intere that were seen in the first map are more clearly defined. Under 'vehicle design' one can see al aspects of the vehicle that the designers have to take and the further down, the constraints that they mest work within. In the second stream there is a break dow of the various areas of research that the team will
look into based on the expertise of the school look into based on the expertise of the school contributing to the project.
He also discusses how the same set of concepts can be configured to any map pattern depending on the context of the map wich plays an important role in map. As can be seen in Figures 3 and 4, each map consists of the same nodes, but in the first map the importance is given to "living thing" and what tha concept entails, while in the second map the emphasis is placed on the "molecule" [7]

This is very useful for engineering design a it gives the team flexibility in situations where the constraint to another. The designers can reconfigure the map without having to start from the beginning.

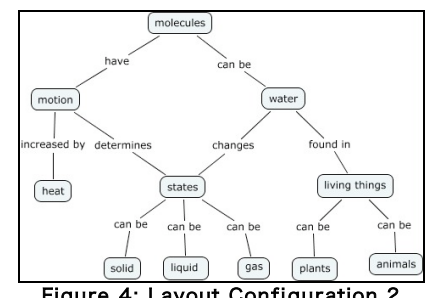

Figure 4: Layout Configuration 2
A study of concept maps in design done by the University of Massachusetts shows that they at's cradle. Linear patterns represent a step-byrelationship from one concept to another, but showed no interrelation between a number of concepts or need to iterate the map to include new concepts. While the cat's cradle, branched and web patterns al showed more diverse connections between concepts. cing [5]. Looking back to Figure 2, even though it has evolved to show more information, the layout of the map does little to allow for faster assimilation of the knowledge that it represents. By following the link to each concept, there is a definite hierarchical pattern between the two streams, thus leading to the distinction, in terms of layout, of the two major concepts as they are spaced out and have their subsystems clearly organized under them. The 'vehicle/mechanism to facilitate research' is noticeably seen as the link between two concepts.

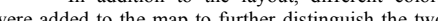
areas of interest, thus allowing the viewer to immediately see all the concepts relating to the vehicle design and all the ones relating to research.

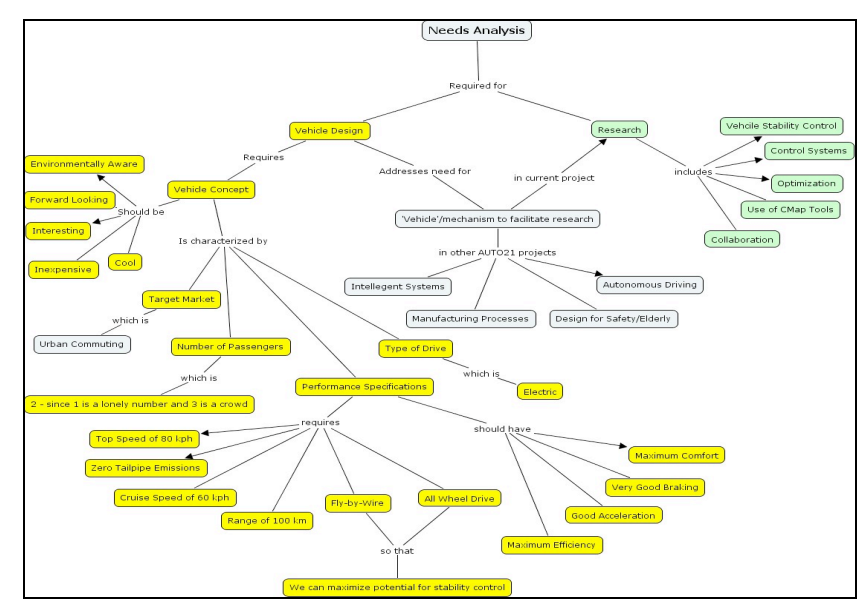

Figure 5: Project Concept Map with Layout Editions 


\subsection{Concept Map Linking}

As more knowledge is added to a map they ineviably become very expansive and require information that the map contains. This retards the effectiveness of the map since there is now too much information in the one map to be gleamed at firs glance. A NASA/HMC collaborative study [1] howed that a map has to be able to fit in one screen usefulness. To add more information major concept in the main map should link to minor maps tha contain more details about the subject of the major concept. This allows for the main map to be a guide to the information while the minor maps can go in more detail about each topic.

major maps, see Figure 6 , shows requirements and constraints that the princip investigators had placed on the design. Upon closer inspection of this map there was a lot of informatio here that had been repeated from the previous map showing the evolution of the project (Figure 5). Thr show new knowledge in the project. To solve this problem, Figure 5 was iterated so that the concept a node 'Performance Specification' now contained link to the map with all the design limitations, Figur 7. This makes the "project" map more conducive quick knowledge capture as it reduces the number of which shows a greater understanding of how the knowledge is interconnected.

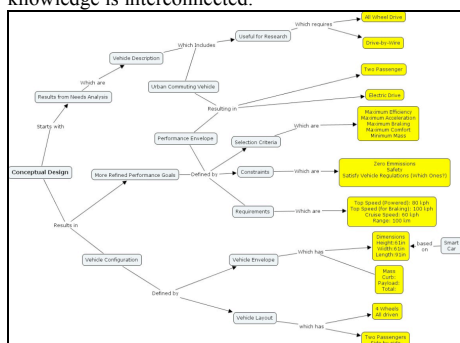

Figure 6: Constraint Map

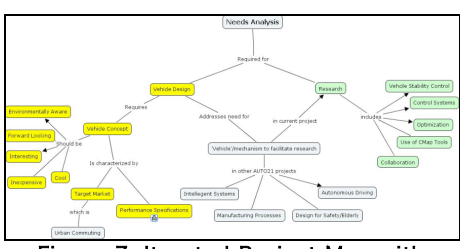

Figure 7: Iterated Project Map with
Linking

3.4 Cyclic Map Pattern

Layout is not limited to hierarchical patterns; there are situations where the flow isn't

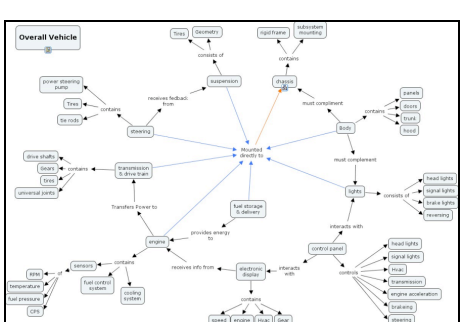

Figure 8: Overall Systems, cyclic pattern

Figure 7 shows a cyclical nature which is necessary to showing how each of the majo subsystems are related to the chassis subsystem. A spread the map over a number of screens making it lose some of its ability to relay information quickly. designers to see how their to be necessary for relates to all the other systems (Figure 8). This can also give an idea as to what magnitude each system is interconnected, providing a sense of priority tha would relate to future design trade off decisions.

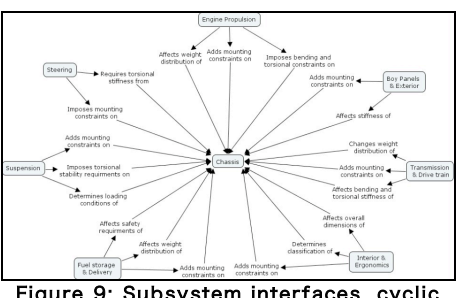

pattern

\subsection{Conclusion}

From the many concept maps that have been shown in this report, there is a clear progression in the amount of information that each map holds. As the Auto21 project has developed the quality of the to layout and style allowing the viewers of the maps the ability to better gleam information. Each time map is changed or more information is added represents iteration in one aspect of the design showing that traditional design methodologies can be a

In using concept maps designers working on common project are better able to organize their the norm of design. By using the oncepts that push of concept maps a design team is not restricted to face-to-face meetings that may be few and far between and a dran on resources, to make any member can provide valuable input from half way around the world either synchronously or

[1] Briggs, Geoffrey et al. Concept Maps Applied to Mars
Exploration Public Outreach.
$I^{\text {st }}$ CMC Exploration Pub

[2] Dumestre, Jeanie. Using CmapTools Software to Assist in Performing Job Task Analysis. CMC CmapTools Syn

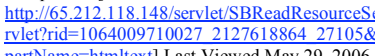

a

King, P.H. and Walker, J.M.T. Concept Mapping
Applied To Design. $2^{\text {nd }}$ Joint EMBSSBMES

[5] Knight, Judith et al. Using Concept Maps to Assess Design Knowledge. 34 A ASEEItiers in Education Conference FIG-10.

[6] Moon, Brian. Concept Maps and Wagon Wheels: Understanding of Team Dynamics. $I^{\text {st }}$ CMC

[7] Novak, Joseph and Gowin, Bob. Learning How to Learn.

ACKNOWLEDGEMENTS

The authors graciously acknowledge the support of

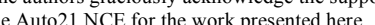

